\title{
Publisher Correction: Social deficits in Shank3-deficient mouse models of autism are rescued by histone deacetylase (HDAC) inhibition
}

Luye Qin, Kaijie Ma, Zi-Jun Wang, Zihua Hu, Emmanuel Matas (D), Jing Wei and Zhen Yan (D)

Correction to: Nature Neuroscience https://doi.org/10.1038/s41593-018-0110-8, published online 12 March 2018.

In the version of this article initially published, the blue diamonds in Fig. $2 \mathrm{a}-\mathrm{d}$ were defined as Shank $3^{+/ \Delta c}+$ saline; the correct definition is Shank $3^{+/ \Delta c}+$ RMD. The error has been corrected in the HTML and PDF versions of the article.

\section{Publisher Correction: Mutations in Vps15 perturb neuronal migration in mice and are associated with neurodevelopmental disease in humans}

Thomas Gstrein, Andrew Edwards, Anna Přistoupilová (D), Ines Leca, Martin Breuss, Sandra Pilat-Carotta, Andi H. Hansen, Ratna Tripathy, Anna K. Traunbauer, Tobias Hochstoeger, Gavril Rosoklija, Marco Repic, Lukas Landler, Viktor Stránecký, Gerhard Dürnberger, Thomas M. Keane, Johannes Zuber, David J. Adams, Jonathan Flint, Tomas Honzik, Marta Gut, Sergi Beltran, Karl Mechtler, Elliott Sherr, Stanislav Kmoch, Ivo Gut and David A. Keays (D)

Correction to: Nature Neuroscience https://doi.org/10.1038/s41593-017-0053-5, published online 8 January 2018.

In the supplementary information PDF originally posted, there were discrepancies from the integrated supplementary information that appeared in the HTML; the former has been corrected as follows. In the legend to Supplementary Fig. 2c, "major organs of the mouse" has been changed to "major organs of the adult mouse." In the legend to Supplementary Fig. 6d,h, "At E14.5 Mbe/Mbe mutants have a smaller percentage of Brdu positive cells in bin 3" has been changed to "At E14.5 Mbe/Mbe mutants have a higher percentage of Brdu positive cells in bin 3."

\section{Publisher Correction: $N^{6}$-methyladenosine RNA modification regulates embryonic neural stem cell self-renewal through histone modifications}

Yang Wang, Yue Li iD, Minghui Yue, Jun Wang, Sandeep Kumar, Robert J. Wechsler-Reya, Zhaolei Zhang, Yuya Ogawa, Manolis Kellis, Gregg Duester (D) and Jing Crystal Zhao (D)

Correction to: Nature Neuroscience https://doi.org/10.1038/s41593-017-0057-1, published online 15 January 2018.

In the version of this article initially published online, there were errors in URLs for www.southernbiotech.com, appearing in Methods sections " $\mathrm{m}^{6} \mathrm{~A}$ dot-blot" and "Western blot analysis." The first two URLs should be https://www.southernbiotech.com/?catno=403005\&type $=$ Polyclonal\#\&panel1-1 and the third should be https://www.southernbiotech.com/?catno=6170-05\&type=Polyclonal. In addition, some Methods URLs for bioz.com, www.abcam.com and www.sysy.com were printed correctly but not properly linked. The errors have been corrected in the PDF and HTML versions of this article. 